\title{
Coeliac disease and type 1 diabetes mellitus: epidemiology, clinical implications and effects of gluten-free diet
}

\author{
A. Marchese $\cdot$ E. Lovati $\cdot$ Federico Biagi $\cdot$ \\ G. R. Corazza
}

Published online: 21 July 2012

(C) Springer Science+Business Media, LLC 2012

Coeliac disease (CD) is a gluten sensitive chronic enteropathy that affects between 1:100 and 1:300 individuals in the general population of the western world [1]. It is characterised by an increased mortality [2]. Autoimmune disorders such as autoimmune thyroiditis, type 1 diabetes (T1DM), Addison's disease, autoimmune liver disease, and Sjogren's syndrome occur much more frequently in patients with $\mathrm{CD}$ than in the general population. The clinical relevance of autoimmunity in coeliac disease is threefold: first, it further deteriorates the clinical course of coeliac disease; second, patients might present only with symptoms of secondary autoimmunity and this could favour the diagnosis of minor coeliac disease; and third, gluten withdrawal could improve the control of some associated autoimmune disorders [3].

The associations between CD and T1DM was recognized over 30 years ago, particularly by paediatricians. The prevalence of $\mathrm{CD}$ among adults and children with T1DM varies in different geographical populations and is observed to range between 1 and $11 \%$ [4-7]. This close association has been ascribed to the same HLA pattern, namely HLA-DQ2 and/or DQ8, which predisposes individuals to both disorders.

Recently, Greco et al. performed a study in the western part of Sicily where they saw that the prevalence of CD among patients with T1DM is $4.5 \%$. They studied 492 subjects (children and adult patients) with T1DM who had been followed-up regularly at their diabetes unit over a fiveyear period. They found $C D$ in 22 patients [8]. In addition, in accordance with previous studies, they confirmed that $\mathrm{CD}$ is

A. Marchese · E. Lovati · F. Biagi ( $₫)$ - G. R. Corazza First Department of Internal Medicine, Fondazione IRCCS Policlinico San Matteo, University of Pavia, Piazzale Golgi, 19, 27010 Pavia, PV, Italy

e-mail: f.biagi@smatteo.pv.it more prevalent in female subjects with T1DM than in males [5] and that the age of onset of T1DM is younger in patients with double the disease than in those with diabetes only [6].

None of Greco's patients presented gastrointestinal symptoms, only two patients presented extraintestinal symptoms (short stature, anaemia and hyposideraemia) and one female patient developed concurrent herpetiformis dermatitis. In fact, one quarter of CD patients with T1DM are totally asymptomatic but the remainder show gastrointestinal symptoms (such as diarrhoea, anorexia, constipation, vomiting, abdominal distension, pain and malnutrition) or extraintestinal symptoms. The most frequent clues in extraintestinal signs are short stature and iron-deficiency anaemia which are present in approximately $50 \%$ of cases; however, fatigue, pubertal delay and vitamin deficiencies are also common, especially in children. Other systemic signs suggesting CD in T1DM patients are a reduced BMI, diminished mass bone, bleeding due to vitamin K deficiency and raised levels of transaminases [9]. CD symptoms (both gastrointestinal and extraintestinal) seem to be much more frequent in children than in adolescents and adults [10].

In the majority of cases (more than $90 \%$ ), the diagnosis of T1DM precedes that of CD and Greco et al. confirmed this finding. However, some studies reported that T1DM onset can frequently occur in patients already diagnosed with $\mathrm{CD}$ [5]. These data suggest the importance of screening for $C D$ for patients with T1DM at the time of diabetes diagnosis and then annually for several years after diagnosis. In particular, antibody positivity for CD may appear within 6 years from the initial diagnosis of T1DM [4].

Serologial screening for CD consists of endomysial antibodies, antibodies against tissue transglutaminase and deamidated gliadin peptide antibodies. When these antibodies are positive, small intestinal biopsy is mandatory. $\mathrm{CD}$ histology is characterized by different degrees of villous 
atrophy [3]. In T1DM patients, following this screening policy, a high number of subjects with positive CD-related antibodies undergo small bowel biopsy. Although the majority of them show a frank villous atrophy, some of them present only minor alterations, such as an increased number of intraepithelial lymphocyte count. This condition is defined as potential coeliac disease. Most of these patients are symptomless and only a minority $(15.8 \%)$ fail to thrive or show gastrointestinal symptoms [7].

Once diagnosed, CD patients with T1DM should be followed up by means of a periodic check-up in the same guise as CD patients without T1DM. All patients with confirmed CD diagnosis (both symptomatic and silent) on the basis of serological and histological pattern must be treated with a gluten-free diet (GFD) regimen to prevent the increased risk of developing both malignant and nonmalignant complications and, especially for children, to improve weight and height, and to prevent osteoporosis [7].

No definite consensus exists among experts on how to treat potential-CD patients by GFD and no data are available about the follow-up of patients with T1DM and potentialCD. We therefore think that GFD is indicated in those patients with evident symptoms related to gluten-sensitive enteropathy, leaving those who are symptomless on a glutencontaining diet with careful follow-up. In addition, we think that potential-CD patients with T1DM who present numerous episodes of hypoglycaemia could also benefit from GFD. In fact, some studies show that GFD is able to reduce severe hypoglycaemic episodes, well-known for being very dangerous, despite an increase in insulin requirement and levels of $\mathrm{HbA} 1 \mathrm{C}$ that are usually lower at the time of $\mathrm{CD}$ diagnosis $[7,9]$ This fact is probably due to the different formulation and the food-processing procedures used in the manufacturing of gluten-free foods that may elicit a high glucose response although this point is controversial. According to other authors, the inclusion of gluten-free products in the diet of T1DM should not compromise glycaemic control, suggesting a normal glycaemic index of gluten-free products [7].

In our experience we saw at least three patients with uncontrolled long-standing T1DM with several episodes of hypoglycaemia and ketoacidosis that were screened for $\mathrm{CD}$ and found positive for endomysial antibodies. They underwent intestinal biopsy and received a diagnosis of potentialCD. We decided to start GFD in these patients anyway, achieving a considerable improvement in glycaemic control.

Some paediatric studies have also shown a reno-protective effect of a GFD in T1DM [7]. As for microvascular complications, such as nephropathy and retinopathy, data are discordant in adults with CD and T1DM, and this is probably due to the fact that the studies were retrospective with small size samples. Moreover, these patients showed more severe subclinical atherosclerosis as compared with those presenting T1DM or CD only [11].
Finally, we have to remember that GFD is a very demanding diet to maintain on a life-long basis. Adherence to GFD among T1DM-CD patients is generally good in patients with clear clinical symptoms of $\mathrm{CD}$, but poor among asymptomatic patients. For a patient with T1DM, already engaged in coping day by day with a complex chronic disease, the addition of a second 'limiting' condition is probably remarkably difficult. Above all for a child or adolescent, the balance between GFD adherence and daily life is difficult to achieve, and the need to coordinate insulin therapy with proper nutrition and a healthy lifestyle, in order to maintain adequate metabolic control, is already a considerable effort for the young T1DM patient and families [6]. We suggest, however, that the importance of GFD should be stressed for its severe complications such as lymphoma [2], perhaps by ensuring adequate psychological and nutritional support.

Conflict of interest The authors declare that they have no conflict of interest.

\section{References}

1. F. Biagi, C. Klersy, D. Balduzzi, G.R. Corazza, Are we not overestimating the prevalence of coeliac disease in the general population? Ann. Med. 42, 557-561 (2010)

2. F. Biagi, G.R. Corazza, Mortality in celiac disease. Nat. Rev. Gastroenterol. Hepatol. 7, 158-162 (2010)

3. S. Martucci, F. Biagi, A. Di Sabatino, G.R. Corazza, Coeliac disease. Dig. Liver Dis. 34(Suppl. 2), S150-S153 (2002)

4. S. Salardi, U. Volta, S. Zucchini, E. Fiorini, G. Maltoni, B. Vaira, A. Cicognani, Prevalence of Coeliac disease in children with type 1 diabetes mellitus increate in the Mid-1990s: an 18-year longitudinal study based on anti-endomysial antibodies. J. Pediatr. Gastroenterol. Nutr. 46, 612-614 (2008)

5. G. Valerio, L. Maiuri, R. Troncone et al., Severe clinical onset and increate prevalence of other autoimmune diseases in children with celiac disease diagnosed before diabetes mellitus. Diabetologica 45, 1719-1722 (2002)

6. M.E. Camarca, E. Mozzillo, R. Nugnes et al., Celiac disease in type 1 diabetes mellitus. Italian J. Pediatr. 26(38), 10 (2012)

7. U. Volta, F. Tovoli, G. Caio, Clinical and immunological features of celiac disease in patients with type 1 diabetes mellitus. Expert Rev. Gastroenterol. Hepatol. 5(4), 479-487 (2011)

8. D. Greco, M. Pisciotta, F. Gambina et al., Celiac disease in subjects with type 1 diabetes mellitus: a prevalence study in western Sicily (Italy). Endocrine (2012). doi:10.1007/s12020-012-9718-8

9. C. Poulain, C. Johanet, C. Delcroix et al., Prevalence and clinical features of celiac disease in 950 children with type 1 diabetes in France. Diabetes Metab. 33(6), 453-458 (2007)

10. F.H. Mahmud, J.A. Murray, Y.C. Kudva et al., Celiac disease in type 1 diabetes mellitus in North American community: prevalence, serological screening, and clinical features. Mayo Clin. Proc. 80(11), 1429-1434 (2005)

11. S.F. Bakker, M.E. von Blomberg, C.J. Mulder, S. Simsek, Type 1 diabetes and celiac disease in adults: glycemic control and diabetic complications. Acta Diabetol. (2012). doi:10.1007/s00592012-0395-0 